\title{
Particle fuelling experiments with series of pellets in LHD
}

\author{
J. Baldzuhn ${ }^{1}$, H. Damm ${ }^{1}$, A. Dinklage ${ }^{1}$, R. Sakamoto ${ }^{2}$, G. Motojima $^{2}$, R. Yasuhara $^{2}$, K. Ida ${ }^{2}$, \\ H. Yamada ${ }^{2}$, LHD Experiment group ${ }^{2}$, Wendelstein 7-X Team ${ }^{1}$
}

${ }^{1}$ Max-Planck Institut fuer Plasmaphysik IPP, Wendelsteinstr. 1, 17491 Greifswald, Germany
${ }^{2}$ National Institute for Fusion Science, NINS, Toki, Gifu 509-5292, Japan

E-mail: baldzuhn@ipp.mpg.de

\begin{abstract}
Ice pellet injection is performed in the heliotron LHD (Large Helical Device) [1,2]. The pellets are injected in short series with up to 8 individual pellets. Parameter variations are performed for the pellet ice isotopes, the LHD magnetic configurations, the heating scenario, and some others. These experiments are performed in order to find out, whether deeper fuelling can be achieved with series of pellets compared to single pellets. An increase of the fuelling efficiency is expected since pre-cooling of the plasma by the first pellets within a series could help for deeper penetration of the later pellets in the same series. In addition these experiments have to show up, which boundary conditions must be fulfilled to optimize that technique. The high-field side injection of pellets, as proposed for deep fuelling in a tokamak, will not be feasible with the same efficiency in a stellarator or heliotron, because there the magnetic field gradient is smaller than in a tokamak of comparable size. Hence, too shallow pellet fuelling, in particular in a large device or a fusion reactor, will be an issue that can be overcome only by extremely high pellet velocities, or other techniques that will have to be developed in the future. It turned out that the fuelling efficiency can be enhanced by injection of a series of pellets, however further investigations will be needed in order to optimize this approach to deep particle fuelling.
\end{abstract}

[1] O. Motojima, H. Yamada, A. Komori, N. Ohyabu, K. Kawahata, O. Kaneko, S. Masuzaki, A. Ejri, M. Emoto, H. Funaba, et al., Physics of Plasmas, 6 (1999) 1843

[2] M. Wakatani, Stellarator and Heliotron Devices (1998), Oxford University Press, New York, Oxford

Keywords: repetitive pellet injection, particle fuelling, series of pellets

\section{Introduction}

Deep particle fuelling is a permanent challenge, especially in large fusion devices as LHD or the optimized stellarator Wendelstein W7-X [3,4]. In such machines, the large thermodiffusion is enhancing the challenges even further [5]. This holds in particular with regard to a 
future stellarator reactor, where deep tritium fuelling will be required to sustain a stable burning process and to compensate for the loss due to fusion reactions.

Several approaches were investigated, however they will require additional investigation to clarify better their potential for deep particle fuelling [6], and possible restrictions have to be illuminated. The well-developed technique of negative-ion Neutral Beam Injection (NBI), might suffer from too low particle fluxes reaching the plasma center. Also well established is the supersonic gasjet, however this suffers from very shallow particle penetration. Recent developments like the injection of Compact Tori [7] bear potential in that respect but need further development to overcome the drawback of small particle inventory and metallic impurity contamination of the plasma. Finally, conventional gas puff from the plasma edge is the standard technique in all fusion devices. Drawbacks are edge cooling, and the risk to violate (at least in tokamaks) Greenwald edge density limits in the case of too high particle fluxes [8].

Cryogenic pellet injection, providing either hydrogen (H) or deuterium (D) ice pellets at high injection speed, is a promising candidate in present plasma devices. Nevertheless, the specific requirements for pellet injection in future devices, for instance for the core fuelling of ITER [9], will be more demanding and require more sophisticated insight into the process of the ice ablation and the subsequent particle deposition process. This concerns, among other things, the optimization of the pellet injection technique itself. Crucial parameters for deep pellet fuelling are the pellet size and speed, since the pellet mass is limited by restrictions from the boundary of plasma edge cooling. Too strong temperature excursions might trigger instabilities by violating stability limits.

In stellarators, enhanced core radiation could deteriorate the global discharge performance. Strong particle deposition in the plasma edge could result in unwanted particle losses to the vessel wall. This is a critical issue in a reactor, where the tritium inventory and wall deposition have to be controlled in an accurate manner. The tritium fuelling for the burning process will always depend on the external feed and the wall recycling fluxes. But those are determined by the complex interplay with the wall deposition history. The pellet speed should be as high as possible. Boundary-conditions like high repetition rates, a complex injection geometry etc. will make substantial $R \& D$ efforts mandatory to reach the desired pellet velocities well above $1 \mathrm{~km} / \mathrm{s}$.

It was proven that the grad-B plasmoid drift motion during magnetic $\underline{H}$ igh-Field $\underline{\text { Side }}$ (HFS) launch considerably enhances central particle fuelling compared to Low-Field $\underline{\text { Side }}$ (LFS) injection [10]. Mechanical stability of the pellet ice is important for the pellet inward launch into the HFS. HFS normally requires the pellet transfer through curved guiding tubes, in which mechanical and inertial forces impose severe limitations. The optimization of the curve radii of the guiding tubes within the given vessel and coil geometry is therefore a crucial task for the lay-out of the pellet injection arrangement. A critical drawback for the concept of HFS in stellarators is the small value of $\operatorname{grad}-B$ (for instance about $0.8 \mathrm{~T} / \mathrm{m}$ in $\mathrm{W} 7-\mathrm{X}$ ) compared to a tokamak with about $2 \mathrm{~T} / \mathrm{m}$. Future pellet experiments in $\mathrm{W} 7-\mathrm{X}$ will be conducted to exploit the potential and limitations of HFS compared to LFS.

Deep particle fuelling by series of pellets (even with only shallow penetration) instead of single pellets motivated the experiments described in this paper. The specific question 
addressed in this study is to examine whether a series of pellets is useful for effective core fuelling. The idea is that earlier pellets might help later pellets within that series to penetrate deeper and to fuel better, for instance by pre-cooling of the plasma within the ablation and deposition zone.

The pellet injection experiments in LHD are performed for a variety of pellet and plasma parameters to figure out, which of these parameters are most suitable to optimize deep fuelling, avoiding at the same time too strong plasma cooling, or approaching too close to Electron $\underline{\text { Cyclotron }}$ Resonance (ECRH) Heating cut-off densities.

This paper is organized as follows: first the realization of the experiments, the chosen discharge conditions, and the shot-to-shot modifications of the experimental conditions are described. The experimental results are presented for a compilation of shots, and the impact of the individual pellet parameters on the fuelling efficiency and some plasma parameters are investigated. Finally the findings are discussed with regard to future pellet experiments in W7-X.

\section{Experiment}

The LHD pipe-gun system [11] allows for a variation of the pellet material (H or D), the pellet repetition frequency and the pellet size. The pellets are always injected from the low field side. As working isotope species of the background plasma, deuterium and hydrogen are used. Series of pellets of variable lengths and ice isotopes are injected, and systematic variations of the plasma heating method and the major axis position are performed to investigate the role of the background plasma.

The pellet speed and the plasma starting density prior to the pellet injection are kept constant, as well as the total heating power. Throughout all pellet injection discharges performed, it was attempted to maintain stable and reproducible shot-to-shot conditions. However, due to the variation in heating devices, magnetic axis position, pellet number per shot etc. it could not be avoided that variations in the discharge conditions occurred. Additional variability of the discharges on the shot-to-shot basis is induced by the impact of long series of pellets with enhanced particle load to the vessel wall. This modifies the particle inventory for later discharges in a hardly predictable manner by wall recycling fluxes. Finally, slight variations in the plasma heating power, the jitter in pellet size and velocity, the additional influx of impurities from the wall (as a function of all the above mentioned parameters) increase the spreading of experimental conditions.

In order to compile all attained results in a conclusive manner, global discharge parameters are compared with each other on a basis of the above described variations. Decisive for central pellet fuelling is the fuelling efficiency (defined as the number of all particles in the pellets compared to the finally deposited particles in the plasma), the global increase of plasma particles after pellet injection, the maximum number of pellets within a series of pellets without deteriorating impact on the target plasma. This includes also the boundary conditions imposed by the heating cut-off densities or the effect of the so-called temperature hole in LHD [12], which appeared during the injection of very long series of pellets. 
On LHD a 20-barrel pipe-gun system provides up to 20 pellets. Each pipe can be charged with ice and can be operated independently from the others. This allows for the simultaneous injection of $\mathrm{H}$ and $\mathrm{D}$ ice. As pellet repetition frequencies $30 \mathrm{~Hz}, 90 \mathrm{~Hz}$ and $300 \mathrm{~Hz}$ were chosen. The pellet speed was kept constant at about $1000 \mathrm{~m} / \mathrm{s}$ with a jitter of typically $+/-50$ $\mathrm{m} / \mathrm{s}$. Different pipe diameters allow for the formation of cylindrical pellets with either $3 \mathrm{~mm}$ or $3.4 \mathrm{~mm}$ diameter. The pellet mass (i.e. the injected particle amount) is calculated from the ice density and the diameter of the pipe. The hydrogen pellets contain $1.11 \cdot 10^{21}$ particles ( 3 $\mathrm{mm})$ and $1.62 \cdot 10^{21}$ particles $(3.4 \mathrm{~mm})$. The deuterium pellets contain $1.27 \cdot 10^{21}$ particles ( 3 $\mathrm{mm})$ and $1.85 \cdot 10^{21}$ particles $(3.4 \mathrm{~mm})$. As no microwave cavity for the measurement of the pellet mass in-flight is available, it is assumed that all pellet particles are deposited in the plasma. In addition, the distance between the pellet injector and LHD is short $(\approx 3 \mathrm{~m})$ therefore the loss of pellet mass inside the guiding tube by erosion is neglected. For the calculations and considerations in the following chapters, where the pellet mass plays a role, we restrict ourselves only to a (linear) correlation between the quantities. A possible loss of pellet mass, for instance by erosion in the guiding tube, is therefore considered as a systematic effect which acts on all pellets in the same manner and will not affect the global conclusion that is drawn from the correlation.

The length of series of pellets was varied between one and eight pellets. However, it turned out that only series up to four pellets could be performed in a stable and reliable manner. Longer series of pellets mostly finished in un-predictable plasma scenarios, terminated discharges or temperature-hole discharges. In practice, series of 1, 2 and 4 pellets were used for the numerical analysis below. In a few cases, also discharges with 6 or even 8 pellets were stable enough for an analysis. However, these discharges could not be reproduced.

Fast photodiodes, partly equipped with front filters for different spectral lines, deliver the exact time of pellet penetration as well as the ablation time duration. For the evaluations described below the broadband signals without filter have been used. For optical survey of the ablation, fast video cameras are used. Thus it can be assessed whether individual pellets are broken or intact.

The plasma discharges are heated by a combination of ECRH and both positive and negative ion NBI, or NBI only. The starting central electron density prior to series of pellets is kept at about $\approx 1 \cdot 10^{19} \mathrm{~m}^{-3}-3 \cdot 10^{19} \mathrm{~m}^{-3}$, this value albeit being slightly dependent on the achievable heating devices and the wall conditioning. That heating combination is used to maintain stable density conditions throughout the discharges. For the ECRH typically a heating power of up to $\approx 2.8 \mathrm{MW}$ is used. For the NBI, three beamlines with negative ion sources are available with a nominal output power of $5 \mathrm{MW}$ each, and two beamlines with positive ions and up to 7 MW nominal output power each. The five beamlines are partly operated with hydrogen, partly with deuterium. The two positive beamlines (operated with deuterium) are also used for

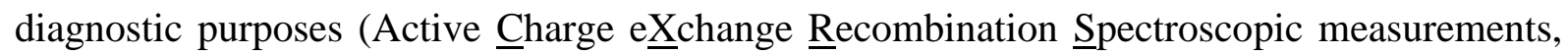
CXRS) [13].

The average magnetic axis position in LHD is varied between $3.6 \mathrm{~m}, 3.75 \mathrm{~m}$ and $3.9 \mathrm{~m}$, resulting in slightly different heating power deposition profiles, power load to the vessel wall and wall recycling conditions. Due to the recent deuterium campaign in LHD, the majority of shots is performed in deuterium as working gas. Only at the end of the deuterium campaign, a 
short hydrogen campaign followed for comparison purposes with hydrogen as working gas. According to CXRS measurements, the ratio $\mathrm{H} /(\mathrm{H}+\mathrm{D})$ is typically in the range $\approx 0.6$ for the hydrogen discharges and $\mathrm{D} /(\mathrm{H}+\mathrm{D})>0.9$ for the deuterium discharges.

Key diagnostics for our experiments is Thomson scattering [14], providing profiles of electron density $\mathrm{n}_{\mathrm{e}}$ and electron temperature $\mathrm{T}_{\mathrm{e}}$ with a laser repetition frequency of $30 \mathrm{~Hz}$. As the laser frequency is smaller than the actual pellet repetition frequency (or equal for the case of 30 $\mathrm{Hz}$ ), the Thomson profiles are numerically interpolated in effective radii and time by cubic spline interpolation. This provides $n_{e}$ and $T_{e}$ values exactly at the points in time when pellets were injected.

In a first step of the numerical data evaluation, the Thomson raw profiles are cross calibrated using the absolutely calibrated Far Infrared Interferometer data. Obvious outliers are ignored to improve the later data interpolation and fitting procedures. After the cubic spline interpolation of $\mathrm{n}_{\mathrm{e}}\left(\mathrm{r}_{\mathrm{eff}}, \mathrm{t}\right)$ the time traces of the total particle content of the plasma are calculated. For the calculation of the global plasma volume and the particle content inside the

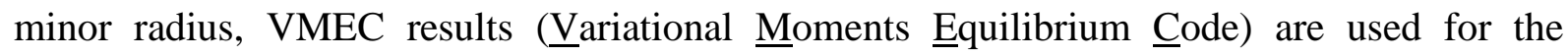
position of the magnetic axis and the size of the real minor radius $a$ as a function of the plasma beta. To minimize possible errors introduced by the particle transport of the background plasma during the series of pellets, the Thomson density profiles are integrated over the minor radius in a very short time distance before the first and after the last pellet of each series; typically within 1-2 msec. The impact of density changes of the background plasma after the series of pellets are therefore minimized. However, the grad- $B$ drift induced effects on the pellet deposition will be included by the choice of this timescale, because this post-pellet particle drifts are essential for the final deposition of the material in the plasma [15]. If pellet particles are lost immediately during the post-pellet phase, for instance because the grad-B drift is directed outward in the experiment, this will be included in the data for our study below.

For our study exactly 100 discharges can be used. More discharges had been performed, however failures in the shot series (premature heating power-off, unwanted plasma collapses etc.) made some of them useless for us.

\section{Results}

Into the 100 discharges that are used for the investigations, 251 pellets had been injected. In some of the plots below, fit lines are included. These serve only for the purpose to show the rough trend of the data and to guide the eye. The scatter of the data points, which is in some cases rather large, is due to the fact that all above mentioned parameters are varied. To investigate the impact of individual parameters, the 100 discharge cases are therefore ordered according to the heating method, the pellet material isotope, the pellet repetition frequency and the major axis position.

These four results are shown in the figures 1-4, where always the total particle increase in the plasma due to pellet injection (x-axis) as a function of the pellet fuelling efficiency (y-axis) is given. The particle increase is calculated from the difference of the Thomson scattering $n_{e}$ 
profiles before and after the pellet (series), after integration over the entire minor plasma radius. That particle increase is therefore a measure for all particles that are finally deposited in the plasma after pellet injection, either for single pellets or series of pellets. The fuelling efficiency is the ratio of all deposited particles in the plasma, divided by all particles provided by the pellets. For each discharge, the fuelling efficiency and the total particle increase are calculated from the measured Thomson data after the mentioned cubic spline interpolation. Then each discharge obtains a label indicating the heating method, the pellet ice isotope, the pellet repetition frequency and the LHD magnetic axis position. Then the 100 discharges (each data point corresponds to one discharge) are plotted together as scatter plots, each label depicted as another symbol (stars, crosses or circles).

It has to be mentioned that along both axes of the plots variables are included that are not completely independent from each other. This is either the particle increase in the plasma, or the number of particles summarized over all pellets. Either the one or the other will always appear directly as the parameter along the one axis, and as a hidden constraint in the parameter along the other. Decisive for the final regression is the actual deposition of the pellet particles in the plasma with a certain efficiency, and this is chosen as the parameter along the y-axis.

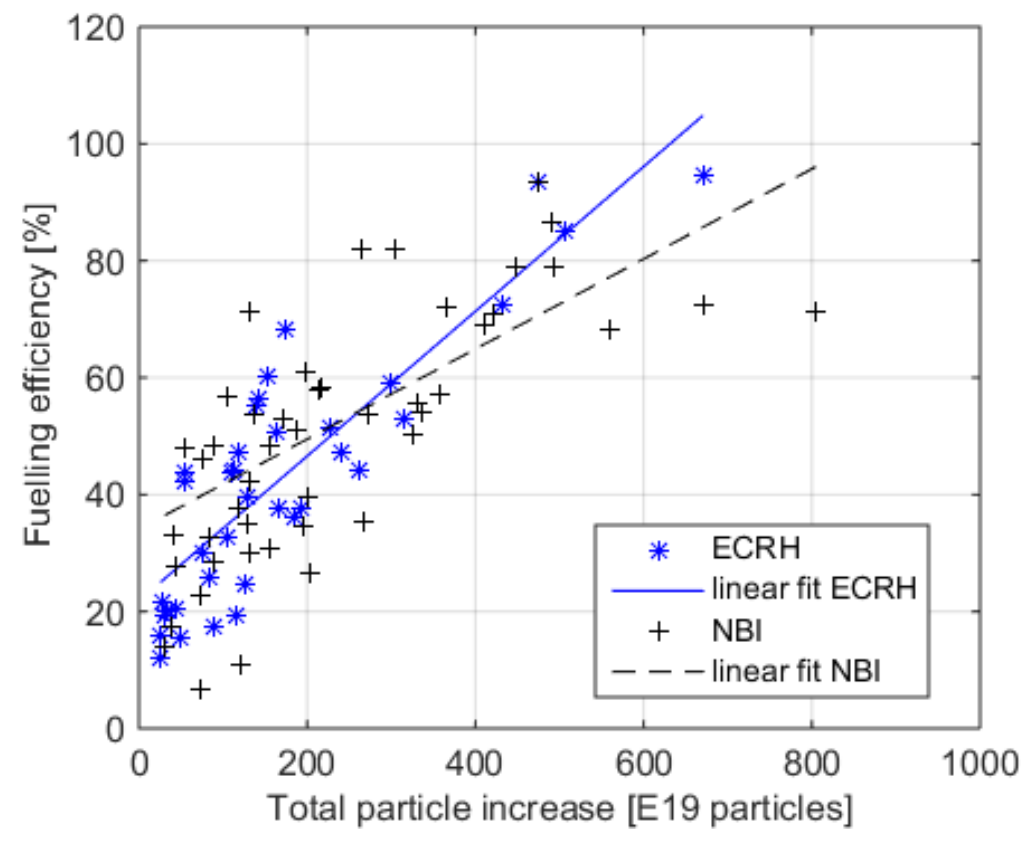

Figure 1. Pellet fuelling efficiency after pellet injection (y-axis) versus the total particle increase (x-axis) for two heating scenario: ECRH combined with NBI (* symbols, solid line) and pure NBI (+ symbols, broken line). Each data point represents one series of pellets into one plasma discharge. The two lines show the result of a linear fit to the corresponding data points.

Fig. 1 shows the results for the comparison between discharges heated by NBI only and the combination of ECRH and NBI. For both heating cases, an increase of the fuelling efficiency 
can be observed if the number of deposited particles in the plasma is increased. This means that longer series of pellets in fact enhance the fuelling efficiency compared to smaller series or single pellets. The fitted lines show a slightly stronger increase of the fuelling efficiency for the case of ECRH plus NBI, however the scatter of all data points is larger than the deviation between the two heating methods and is therefore not significant. The final deposition of pellet particles does therefore not depend on the heating method, at least not for the two methods employed here, since it could be suspected that supra-thermal electrons, produced by the ECRH, will play a role for enhanced ablation closer to the plasma edge and subsequent poorer fuelling efficiency.

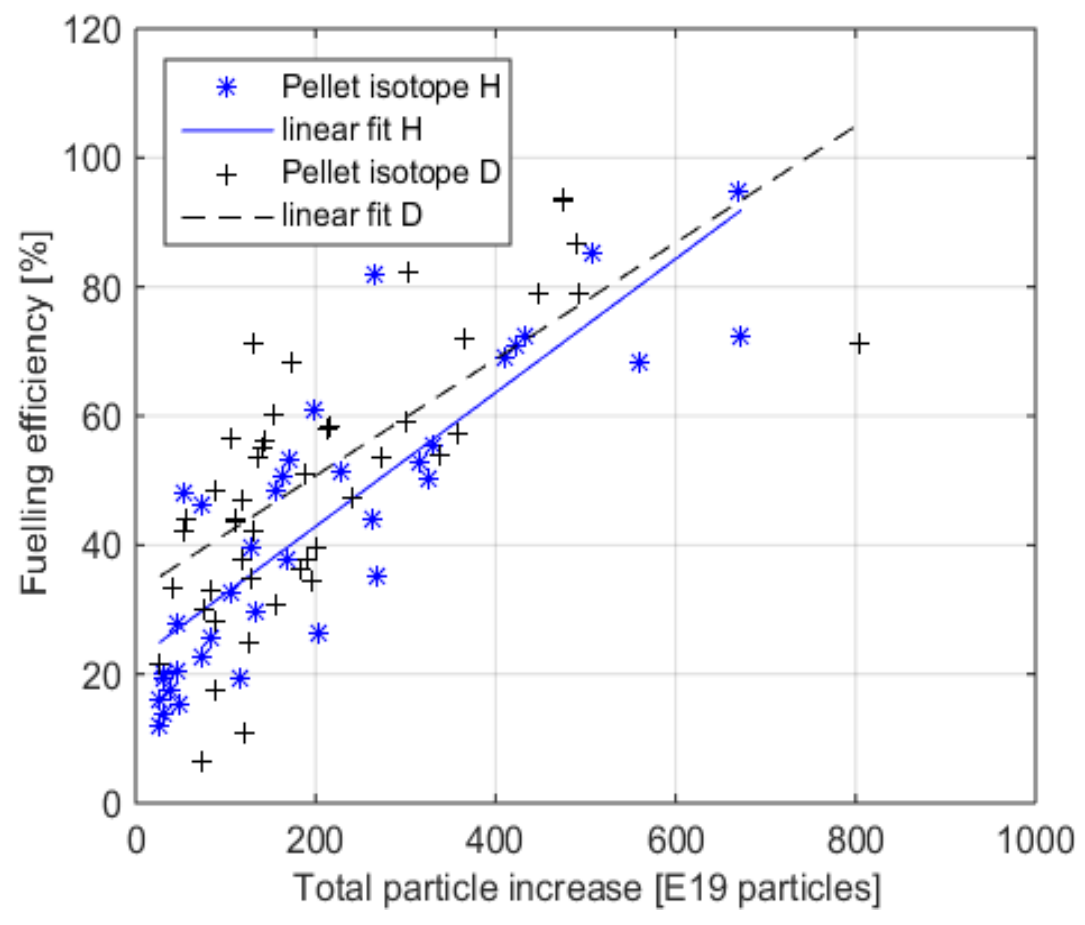

Figure 2. Pellet fuelling efficiency after pellet injection (y-axis) versus the total particle increase (x-axis) for two pellet material isotopes: hydrogen $H(*$ symbols, solid line) and deuterium ice $D(+$ symbols, broken line) pellets. Each data point represents one series of pellets into one plasma discharge. The two lines show the result of a linear fit to the corresponding data points.

Fig. 2 shows the result of the comparison between hydrogen $(\mathrm{H})$ and deuterium (D) ice pellets. Again, for both cases an increase of the fuelling efficiency can be observed if the number of deposited particles in the plasma is increased. The fitted lines lie close to each other, indicating that the pellet ice isotope plays no significant role for the deposition process, giving hope that also tritium ice (or mixtures of isotopes) might behave in a similar way, i.e. that longer series of pellets might be more efficient for deep fuelling than single pellets or shorter series of pellets. This could therefore make this method also for tritium fuelling suitable. 


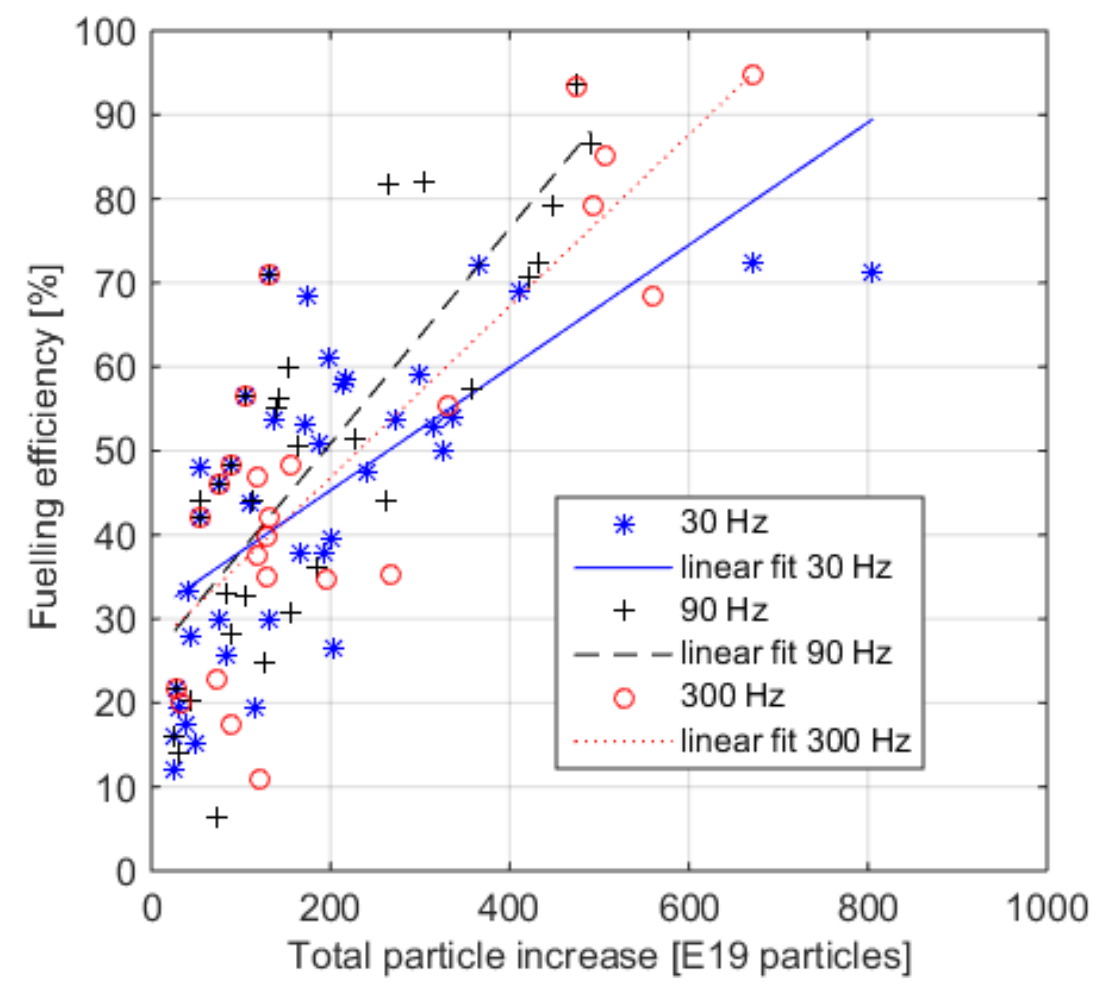

Figure 3. Pellet fuelling efficiency after pellet injection (y-axis) versus the total particle increase (x-axis) for three pellet repetition frequencies within each series of pellets: $30 \mathrm{~Hz}$ (* symbols, solid line), $90 \mathrm{~Hz}$ (+ symbols, broken line) and $300 \mathrm{~Hz}$ (o symbols, dotted line). Each data point represents one series of pellets into one plasma discharge. The three lines show the result of a linear fit to the corresponding data points.

Fig. 3 shows the result of the comparison between three cases of a pellet repetition frequency of $30 \mathrm{~Hz}, 90 \mathrm{~Hz}$ and $300 \mathrm{~Hz}$. Again the three fitted lines lie close to each other, closer than the scatter of the individual data points. This indicates that the time interval between the pellets within the series plays no quantitative role within the ensemble of performed experiments. If the electron temperature is lower when the time distance between the pellets is smaller, this effect might become more pronounced. Nevertheless, even in the lowest frequency case (30 $\mathrm{Hz}$ ) each later pellet in the series experienced a lower $\mathrm{T}_{\mathrm{e}}$ compared to earlier pellets in the series, supporting the concept of pellet pre-cooling within a series. Why the impact of the repetition frequency is not clearer, however, is hard to understand. We can only speculate that all effects that determine the final fuelling efficiency occur on time scales faster than $300 \mathrm{~Hz}$ $(3.33 \mathrm{~ms})$ and are therefore for all three frequencies chosen $(30 \mathrm{~Hz}, 90 \mathrm{~Hz}, 300 \mathrm{~Hz})$ of the same relevance. One example for a time resolved sequence is shown in fig. 8 below. 


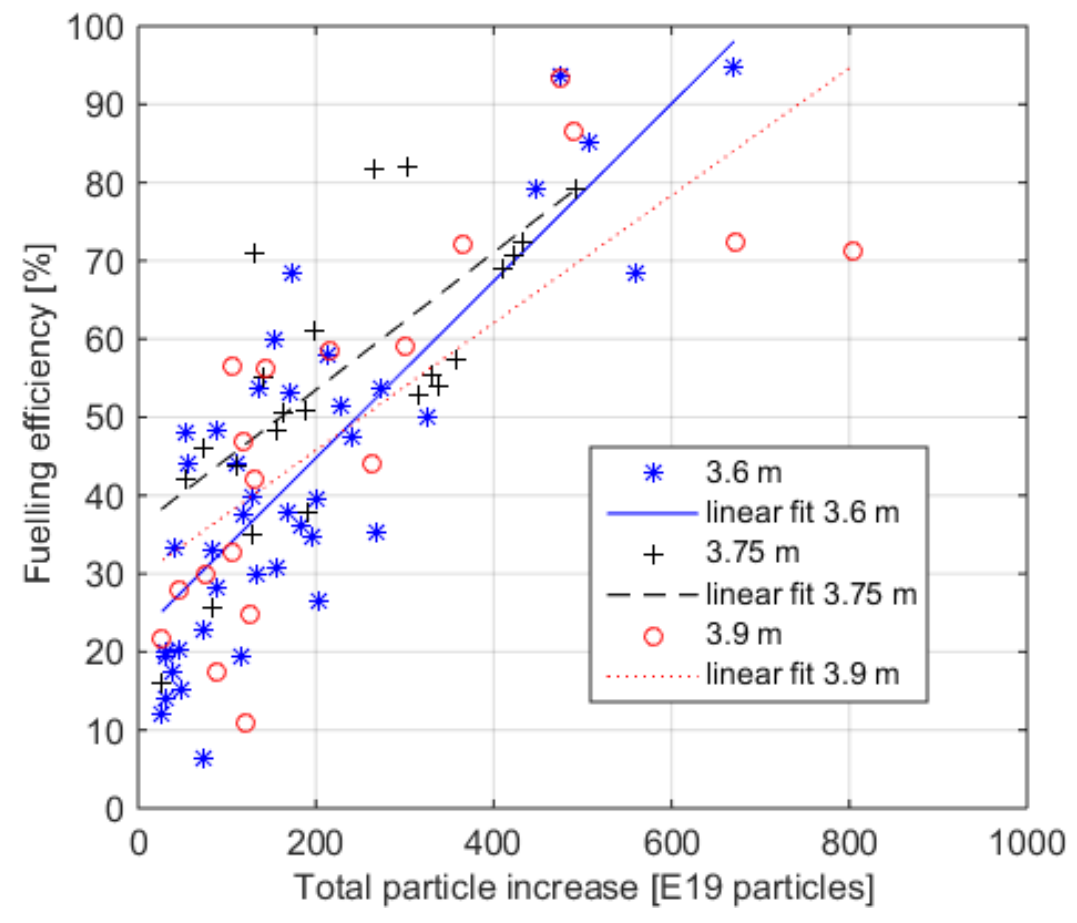

Figure 4. Pellet fuelling efficiency after pellet injection (y-axis) versus the total particle increase (x-axis) for three different positions of the LHD magnetic axis: $3.6 \mathrm{~m}$ (* symbols, solid line), $3.75 \mathrm{~m}$ (+ symbols, broken line) and $3.9 \mathrm{~m}$ (o symbols, dotted line). Each data point represents one series of pellets into one plasma discharge. The three lines show the result of a linear fit to the corresponding data points.

Fig. 4 shows the result of the comparison between three different positions of the magnetic axis in LHD, resulting in different plasma confinement properties [16]. Shown are the cases $3.6 \mathrm{~m}, 3.75 \mathrm{~m}$ and $3.9 \mathrm{~m}$. In addition to different plasma confinement, also the recycling fluxes are supposed to be different because the power load distribution to the vessel wall will differ. But as in the examples before, the three linear lines are so close to each other that any difference is not significant; at least not is a systematic manner. The wall recycling and the global plasma confinement will play a role for the development of each individual discharge. However, neither wall recycling nor global plasma confinement generate significant differences between the discharges. 


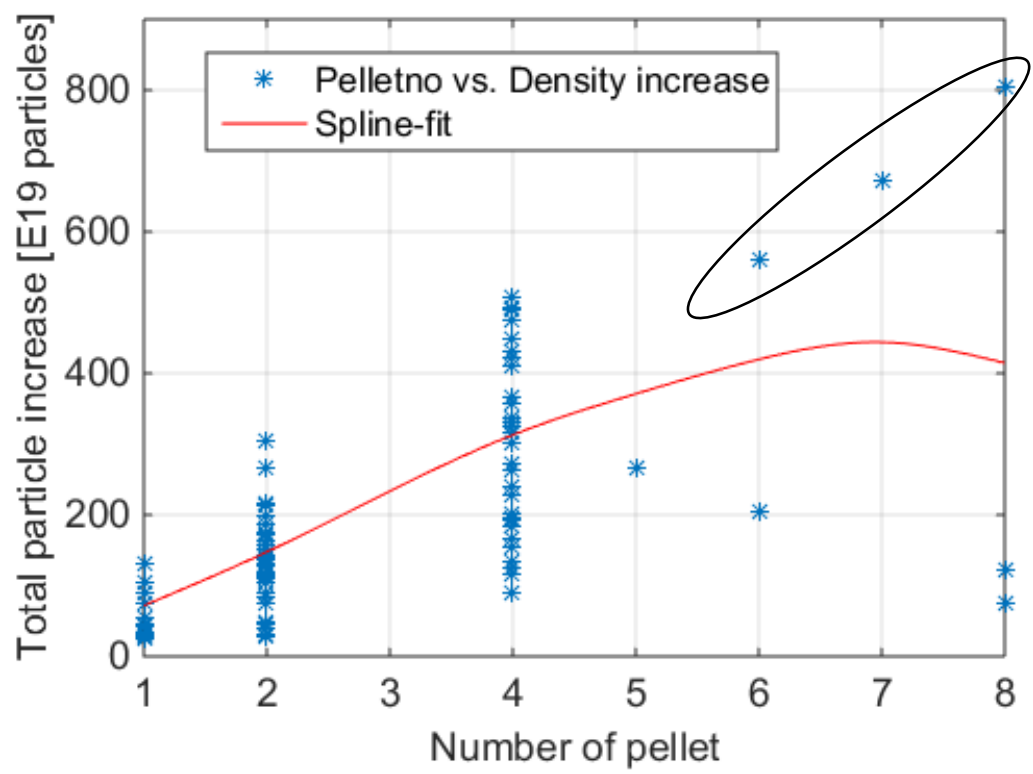

Figure 5. Total particle increase after the series of pellets (y-axis) versus the number of the pellets within one series (x-axis). Each data point (* symbols) represents one series of pellets into one discharge. Regular series of pellets with up to 4 pellets could be performed, only a very few discharges with more than 4 pellets were successful (within the ellipse). The solid line shows a smoothing spline fit to the data points.

Fig. 5 shows the number of pellets within a series (x-axis) versus the total plasma particle increase. The cases with one pellet are the reference cases for the longer series with more than one. The graph shows an increase of the total plasma particle content after pellet injection, if the number of pellets in the series increases. This effect is trivial; however we wanted to investigate whether a saturation effect can be observed if the number of pellets within a series is increased above a certain level. Up to 4 pellets the fitted spline curve is roughly linear, indicating no saturation. For 5 and more pellets, however, the data basis becomes unfortunately too poor for a conclusion. It turned out during the experiments, that most of the discharges terminated prematurely or finished in a temperature hole scenario for more than 4 pellets. The limiting factor might be the available heating power for the densities attained after the series of pellets or the plasma radiated power. Common for these shots is that $T_{e}$ drops to values $<300 \mathrm{eV}$ after the series of pellets; obviously too low to sustain the discharges any longer. Only three of the discharges with 6,7 or 8 pellets could be maintained for a longer time, or they recovered immediately after the series of pellets (lying inside the ellipse in fig. 5). The roughly linear arrangement of these three data point in the plot indicates that the fuelling efficiency for all pellets is comparable, i.e. each further pellet in a series produces roughly the same total particle increase. Due to the small number of these discharges, however, it cannot be concluded which boundary conditions are decisive for either the premature termination or the recovery of a discharge. 


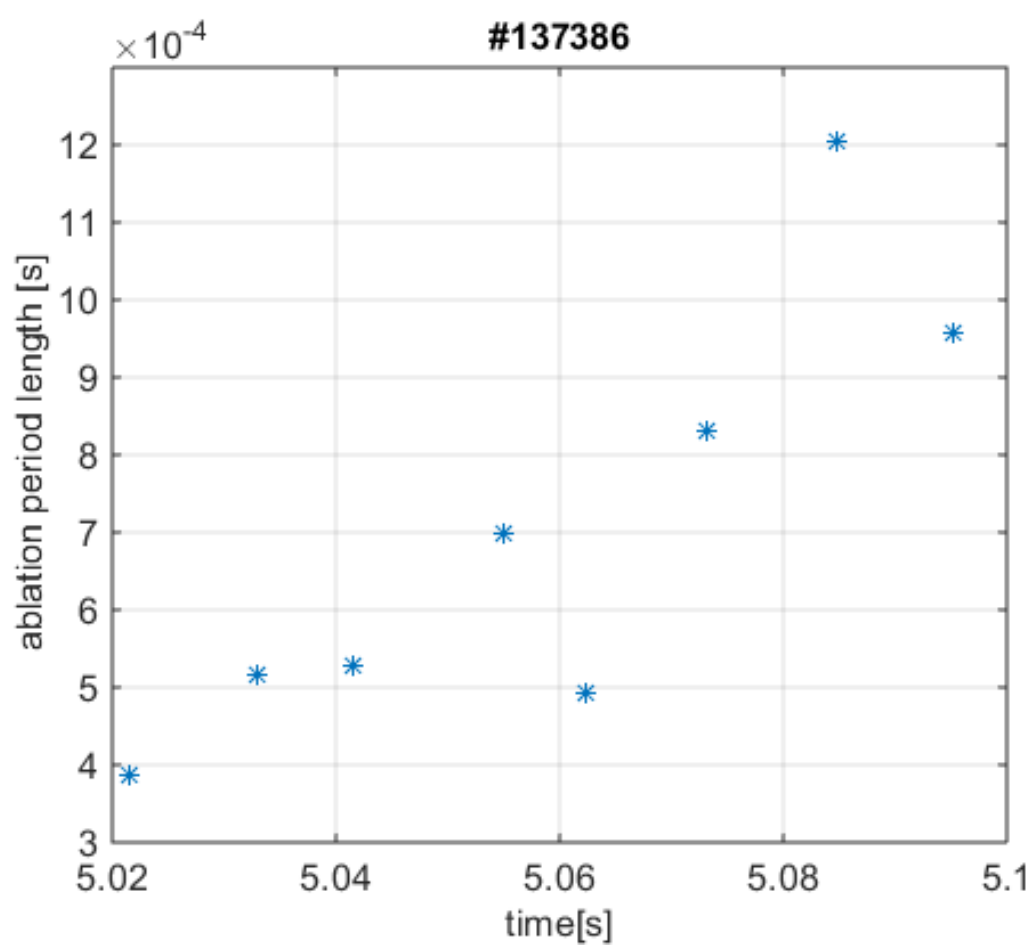

Figure 6. Measured ablation light signal length (y-axis) versus time (x-axis) within one discharge \#137386, for a sequence of 8 pellets. Each symbol represents one pellet. The later pellets show longer light signals than the earlier ones within the sequence.

Fig. 6 shows a typical pellet ablation period length versus the pellet injection time for one discharge with a series of 8 pellets. The later the pellet, the longer is, as a rough trend, the ablation time length. This indicates that later pellets can penetrate deeper, as the pellet mass and the pellet velocity are comparable for the pellets. The first pellets within this series penetrate between $0.39 \mathrm{~m}-0.52 \mathrm{~m}$, while the later pellets can penetrate typically $0.9 \mathrm{~m}-1 \mathrm{~m}$ into the plasma, assuming roughly constant velocity for the pellets. Here it is assumed for simplicity that no bending of the pellet traces, for instance by rocket effects, takes place. Fig. 6 demonstrates the enhanced penetration for later pellets after plasma cooling by earlier pellets. 


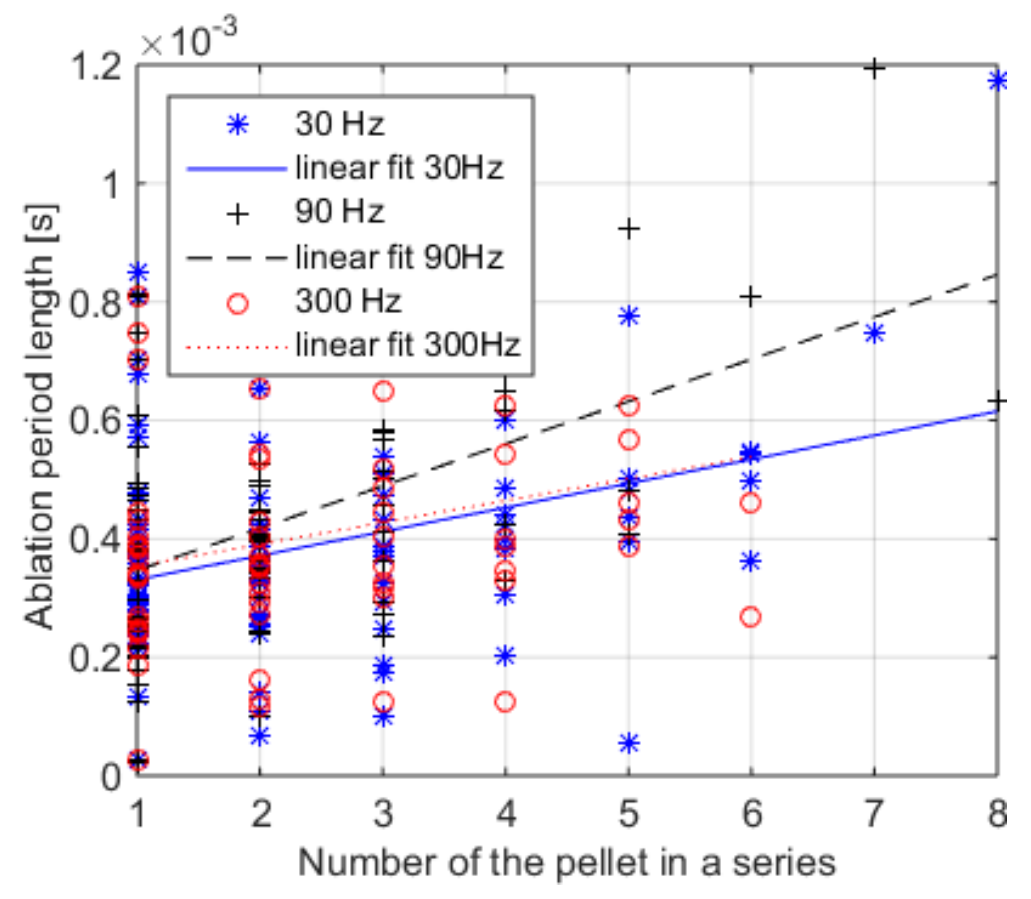

Figure 7. Measured ablation period length (y-axis) versus the number of each individual pellet within a series of pellets (x-axis). Each data point $(*,+$, o symbols) represents one pellet. The longest series were 8 pellets long. The solid lines show the results of linear fits to the data points ( $30 \mathrm{~Hz}$ solid line, $90 \mathrm{~Hz}$ dashed line, $300 \mathrm{~Hz}$ dotted line). Three different pellet repetition frequencies $(30 \mathrm{~Hz} *, 90 \mathrm{~Hz}+300 \mathrm{~Hz}$ o) are distinguished.

Fig. 7 shows the number of each pellet within a series versus the ablation time length for all 251 pellets individually. For each of the three repetition frequencies $(30 \mathrm{~Hz}, 90 \mathrm{~Hz}$ and 300 $\mathrm{Hz})$ a linear fit is performed to the data points. The result show the increase of ablation time (i.e. the ablation depth into the plasma) with the pellet number in a series, as already shown for one example in fig. 6 . The repetition frequency of $90 \mathrm{~Hz}$ seems slightly more favorable to take advantage of the plasma cooling by earlier pellets in a series, compared to $30 \mathrm{~Hz}$ and 300 $\mathrm{Hz}$. However again, the scatter of the data points is too large to draw a sharp conclusion. This supports the earlier finding from fig. 3 that the quantitative time distance between the individual pellets plays no leading role for the exact amount increase in the penetration. 


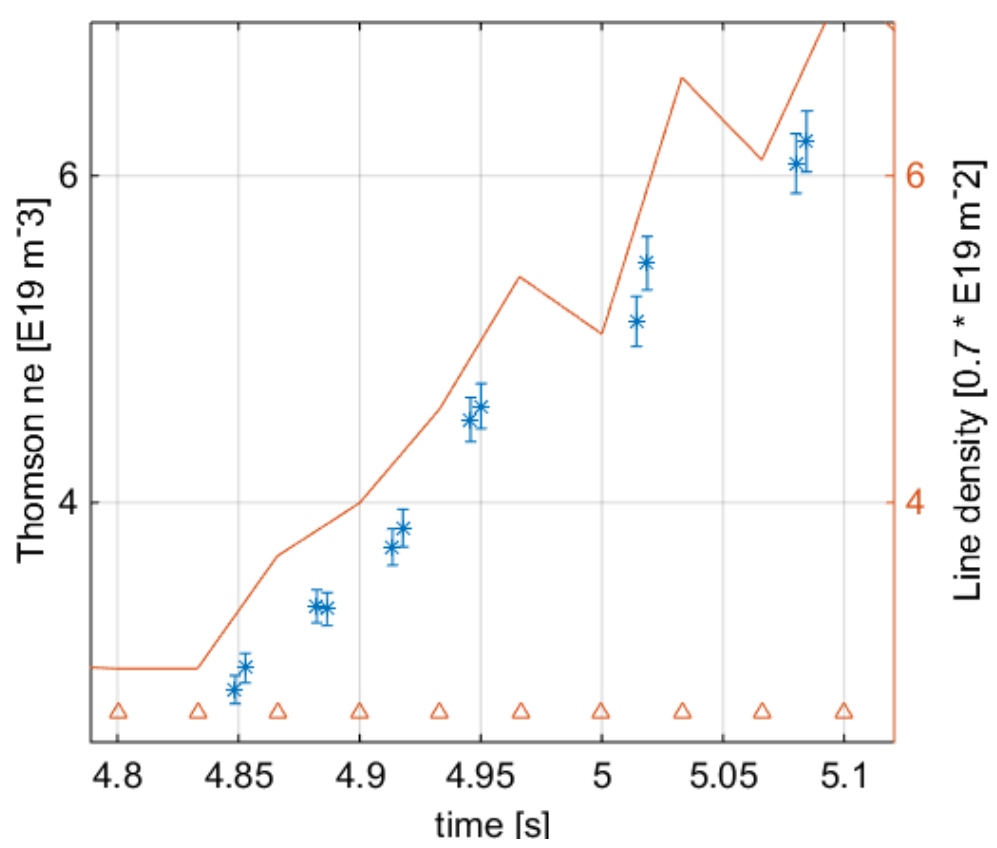

Figure 8. Measured electron density (left y-axis) from Thomson scattering at $r_{\text {eff }}=0.5 \mathrm{~m}$ versus time (x-axis) for one series of 6 pellets into one discharge \# 137413. Each pair of data points (* symbols) shows $n_{e}$ directly before and after a pellet, the error bars indicate typical measurement errors. The triangles show the Thomson laser times for $30 \mathrm{~Hz}$ laser repetition frequency. It is emphasized that each pellet is fired after the same time interval (33.33 ms) after the last Thomson point to make the time bases coherent. The solid line shows the interferometer line density (right y-axis), it's value is roughly matched to $r_{\text {eff }}=0.5 \mathrm{~m}$ by multiplication with an assumed factor of 0.7.

Fig. 8 shows a time sequence of Thomson scattering measurements of $n_{e}(r=0.5 \mathrm{~m})$ for a series of 6 pellets in one discharge. The corresponding data for $T_{e}(r=0.5 \mathrm{~m})$ are shown in fig. 9. This series demonstrates that in fact each pellet experiences a slightly reduced local $\mathrm{T}_{\mathrm{e}}$ after cooling by the earlier pellets in the series, making the above demonstrated deeper penetration possible. The minor radius $r=0.5 \mathrm{~m}$ was chosen at the location where the ablation typically is strongest. Indicated are also the moments of the Thomson laser injection.

This rather long series of pellets with 6 pellets is however not typical with it's monotonically increasing $n_{e}$ and decreasing $T_{e}$. More typical is a rollover behavior, i.e. the increase of $n_{e}$ stops after a certain pellet and decreases for later pellets. Very low $\mathrm{T}_{\mathrm{e}} \approx 200 \mathrm{eV}$ are typical for those discharges. One example is shown in the fig. 10 for $n_{e}(r=0.5 \mathrm{~m})$ and fig. 11 for $\mathrm{T}_{\mathrm{e}}(\mathrm{r}=$ $0.5 \mathrm{~m}$ ) for a series of 6 pellets in one discharge. Obviously the pellets no. 5 and 6 cannot increase the discharge density further, instead it saturates or even decreases slightly for $T_{e}<$ $250 \mathrm{eV}$. That discharge is close to collapse conditions, therefore the line density values deviate strongly from the Thomson data points in fig. 10. 


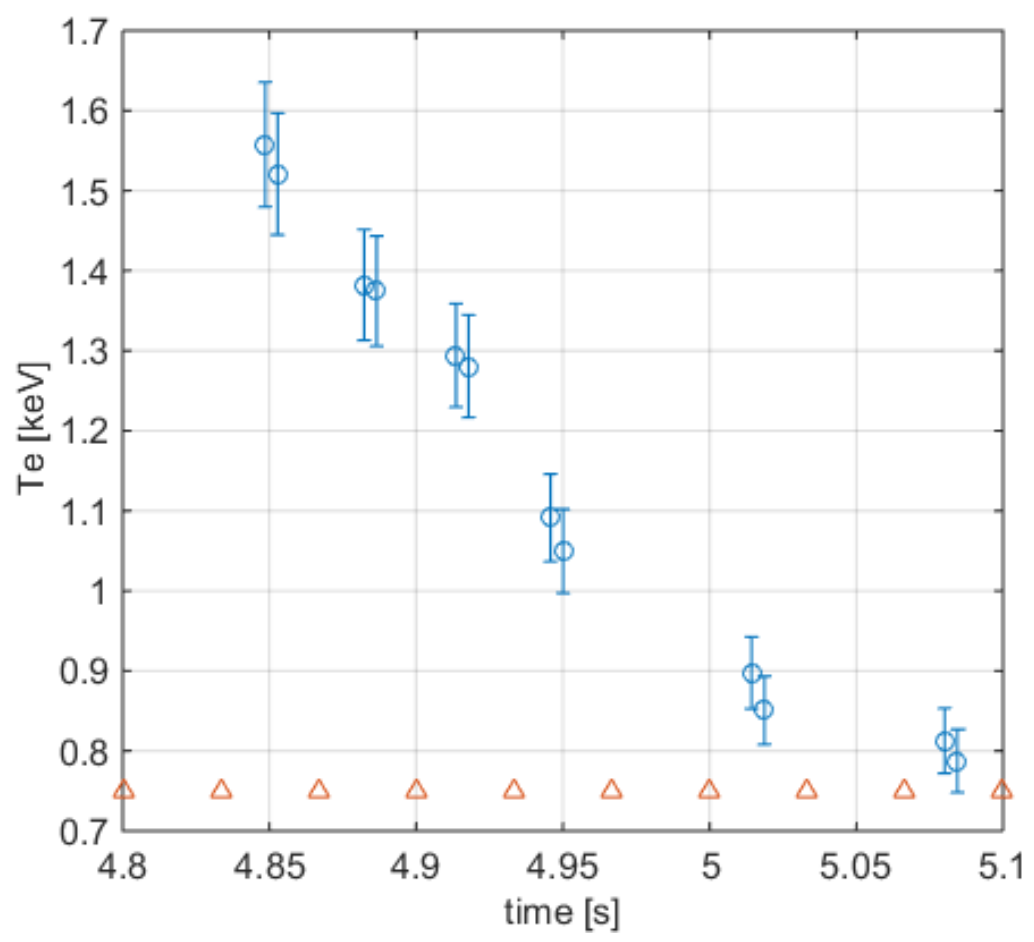

Figure 9. Measured electron temperature (y-axis) from Thomson scattering at $r_{\text {eff }}=0.5 \mathrm{~m}$ versus time (x-axis) for one series of 6 pellets into one discharge, \# 137413. Each pair of data points (circles) shows $T_{e}$ directly before and after a pellet, the error bars indicate typical measurement errors. The triangles show the Thomson laser times for $30 \mathrm{~Hz}$ laser repetition frequency. It is emphasized that each pellet is fired after the same time interval (33.33 ms) after the last Thomson point to make the time bases coherent.

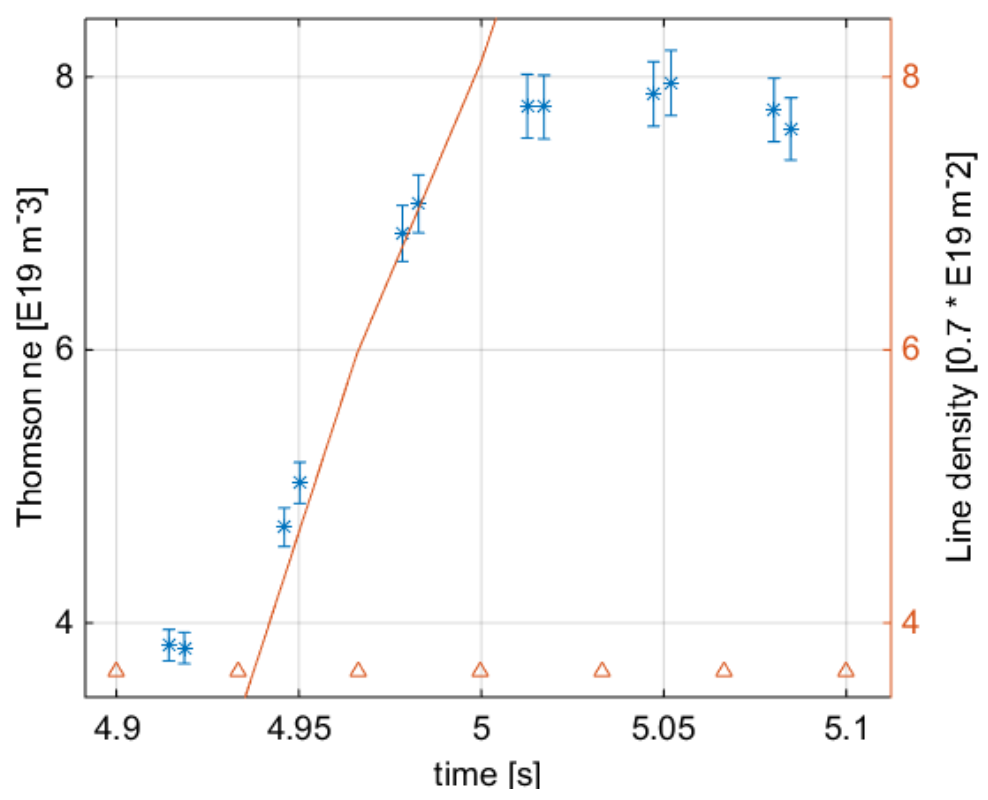


Figure 10. Measured electron density (left y-axis) from Thomson scattering at $r_{\text {eff }}=0.5 \mathrm{~m}$ versus time ( $x$-axis) for one series of 6 pellets into one discharge \# 137453. Each pair of data points (* symbols) shows $n_{e}$ directly before and after a pellet, the error bars indicate typical measurement errors. The triangles show the Thomson laser times for $30 \mathrm{~Hz}$ laser repetition frequency. It is emphasized that each pellet is fired after the same time interval (33.33 ms) after the last Thomson point to make the time bases coherent. The solid line shows the interferometer line density (right y-axis), it's value is roughly matched to $r_{\text {eff }}=0.5 \mathrm{~m}$ by multiplication with an assumed factor of 0.7.That discharge is close to collapse conditions, therefore the line density values deviate strongly from the Thomson data points.

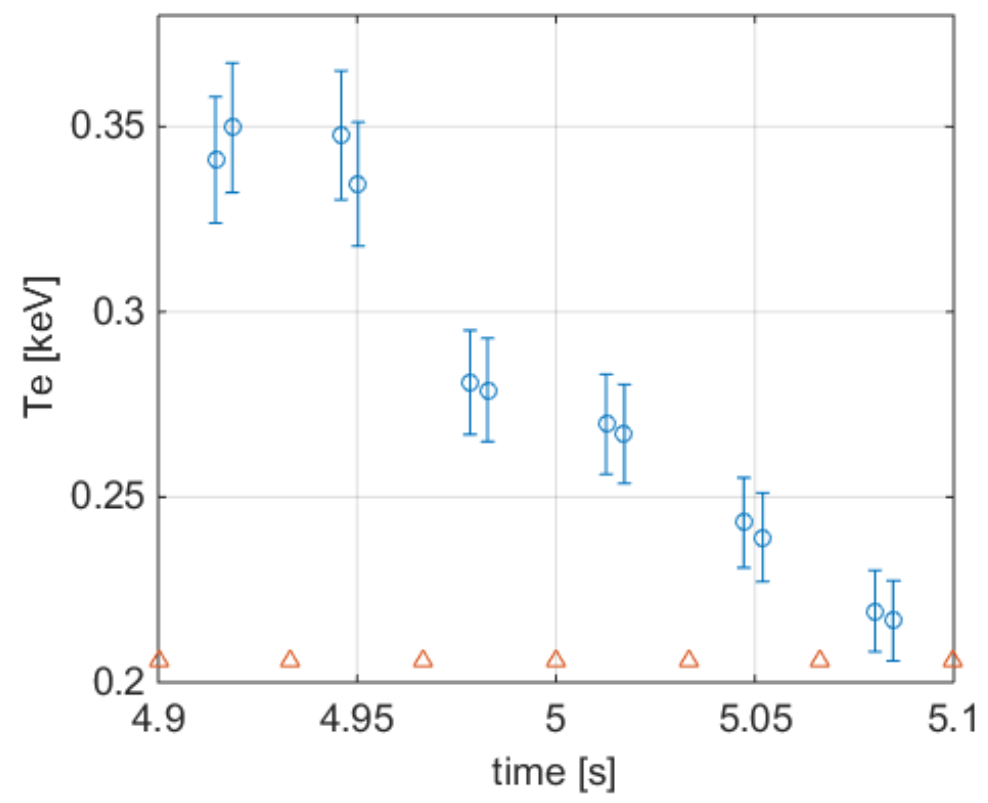

Figure 11. Measured electron temperature (y-axis) from Thomson scattering at $r_{\text {eff }}=0.5 \mathrm{~m}$ versus time (x-axis) for one series of 6 pellets into one discharge, \# 137453. Each pair of data points (circles) shows $T_{e}$ directly before and after a pellet, the error bars indicate typical measurement errors. The triangles show the Thomson laser times for $30 \mathrm{~Hz}$ laser repetition frequency. It is emphasized that each pellet is fired after the same time interval (33.33 ms) after the last Thomson point to make the time bases coherent.

As shown before, the pellet ice isotope obviously plays no role for the effects reported here. The same holds for the plasma ion isotope. The comparison between the pellet results taken during the deuterium campaign and the hydrogen campaign for the plasma working gas shows no considerable difference. This could be due to the fact that during the hydrogen campaign still a certain amount of deuterium was present in the machine. On the other hand, no pure $100 \%$ hydrogen conditions could be maintained during the deuterium campaign. 
To conclude the results: the higher the amount of pellet particles within one series of pellets (or the longer the series), the higher is the overall fuelling efficiency for the entire series of pellets. Boundary conditions like the choice of the heating devices, the pellet material isotope, the pellet repetition frequency or the magnetic configuration play only a minor role for this effect. For the discharge conditions chosen, series of up to 4 pellets can take advantage of the enhanced penetration. Series with 5 or more pellets could not always be sustained reliably. The enhanced fuelling efficiency for a series of pellets is supported by the increased penetration depth for later pellets in a series, compared to earlier ones in the same series. It seems that the cooled flux surfaces after the first pellets will help later pellets in the series to penetrate deeper.

\section{Density profile effects}

To highlight the final deposition of the pellet material in the plasma column, a closer look to the temporal and radial density profile development is mandatory. Below, three prominent examples of the density profile development after the injection of a series of pellets are presented. In all cases a series of pellets of 4 pellets was injected, however with different mechanisms of material deposition inside the plasma column.

A more quantitative treatment of the effects of transient density profile shaping in LHD had been published before [17], also taking into account the mutual interaction between plasma transport as a function of the plasma parameters, and the profile perturbation by pellets. The interplay between pellet re-fuelling and density relaxation phenomena, with focus on the requirements of a burning fusion plasma, is presented in detail in [18]. In order not to repeat the arguments developed there, we restrict ourselves here to the description of the observed phenomena, and their possible implications for the technique of pellet series injection. 


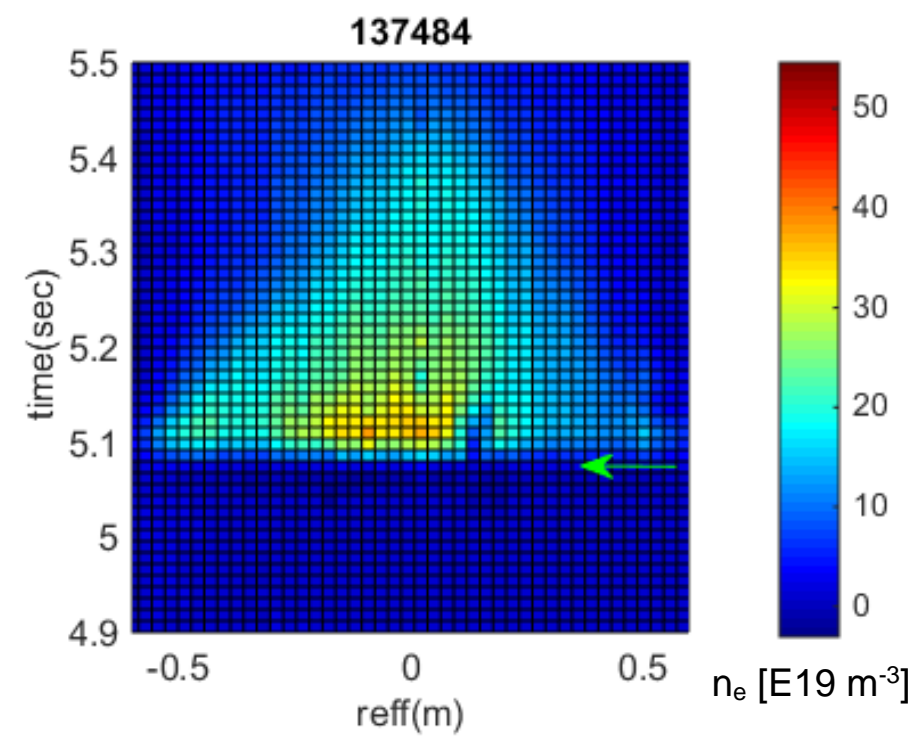

Figure 12. Contour plot of the electron density versus the minor radius ( $x$-axis) and the time (y-axis) for \# 137484. The injection times of the pellets are indicated by an arrow. The side bar indicates the color code of the electron density in units of E19 $\mathrm{m}^{-3}$.

Fig. 12 shows an example of a fast distribution of the pellet material within $<30 \mathrm{~ms}$ even in the plasma center. The pellet repetition frequency was $300 \mathrm{~Hz}$ in this case, resulting in a rather strong cooling of the entire plasma cross section down to several $100 \mathrm{eV}$, supporting deep penetration and central particle deposition. The corresponding electron temperature profiles are shown in fig. 13. Directly after the series of pellets a peaked density profile is realized at low temperature where the central peak density decays away in a time scale of $\approx$ $100 \mathrm{~ms}$, i.e. on a diffusive time scale. In parallel, $\mathrm{T}_{\mathrm{e}}$ recovers adiabatically in the plasma center.

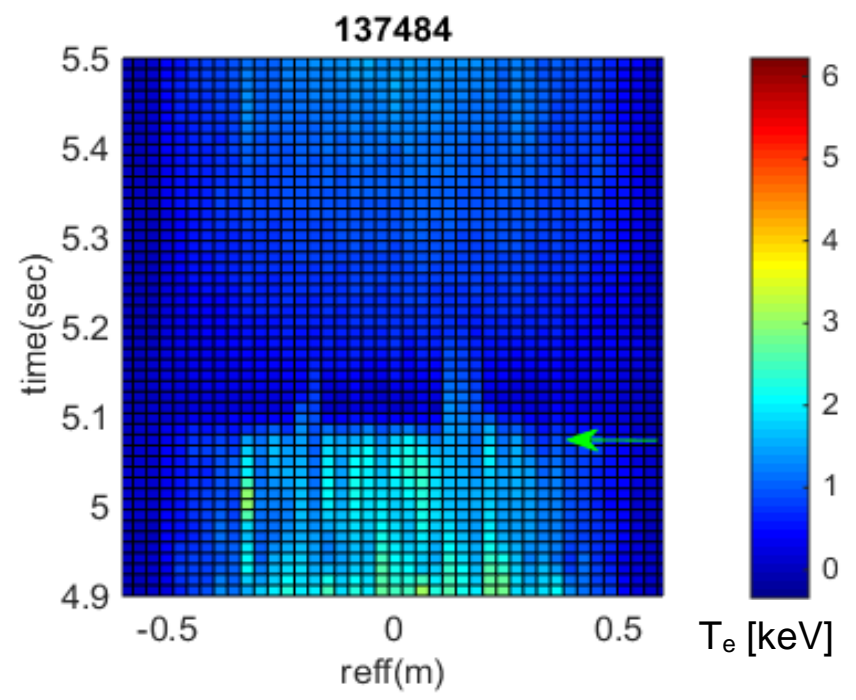

Figure 13. Contour plot of the electron temperature versus the minor radius (x-axis) and the time (y-axis) for \# 137484. The injection times of the pellets are indicated by an arrow. The side bar indicates the color code of the electron temperature in units of keV. 
Within $\approx 10$ milliseconds directly after pellet injection the integrated plasma radiation increases up to $\approx 5.2 \mathrm{MW}$. This considerably high power fraction, compared to the heating power, obviously cools the plasma to a level, where the pellets can penetrate deeply and build up the peaked density profile. It is suspected that this discharge is then close to a premature termination by that excessive cooling. After that phase the radiation level drops down to $<2$ MW (to $<10 \%$ of the nominal heating power). This comparably low radiation level might then help for the subsequent recovery of the discharge. The fast central particle fuelling is of course desirable, however not for the price of such a strong temperature drop as shown in this example. A comparable discharge with 8 pellets instead of 4 recovers only with an extremely hollow density profile after the series of pellets (not shown here).

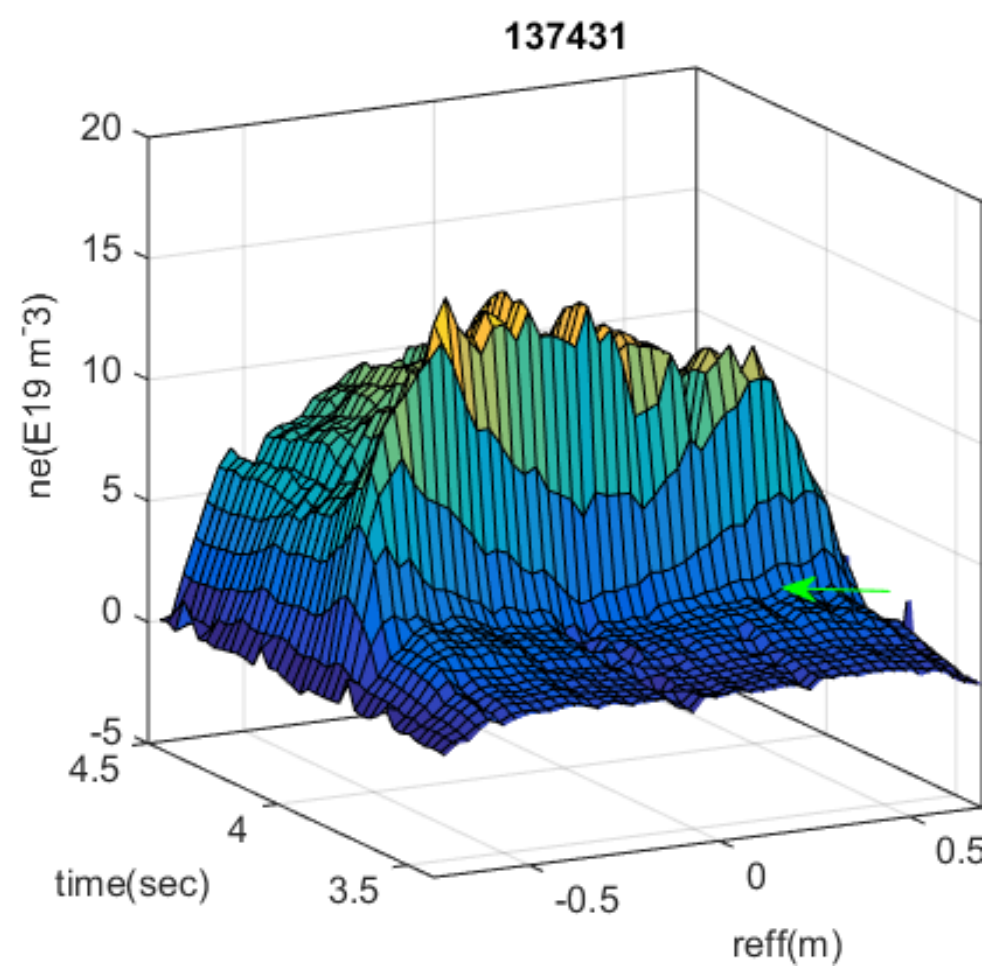

Figure 14. Surface plot of the electron density (z-axis) versus the minor radius (x-axis) and the time (y-axis) for \# 137431. The injection times of the pellets are indicated by an arrow.

An interesting case with 4 pellets, but a repetition frequency of $30 \mathrm{~Hz}$ instead of $300 \mathrm{~Hz}$ is shown in fig. 14. The discharge starts from a slightly hollow density profile, which becomes even more hollow during the series of pellets. However, at the end of the series of pellets, the central density is filled up rapidly within $<30 \mathrm{~ms}$, turning the hollow density profile into a peaked one. Then that profile shape is maintained for several $100 \mathrm{~ms}$, while the central $n_{\mathrm{e}}$ decreases on a diffusive time scale. The slight left-right asymmetry of the density values is caused by a mis-alignment between the laser trajectory through the plasma and the optical imaging onto the detector array. The corresponding $T_{e}$ profiles are shown in fig. 15. The central $\mathrm{T}_{\mathrm{e}}$ decreases in the phase prior to the pellet injection (between 3.4 and $3.8 \mathrm{~s}$ ) because the density is ramped up. 


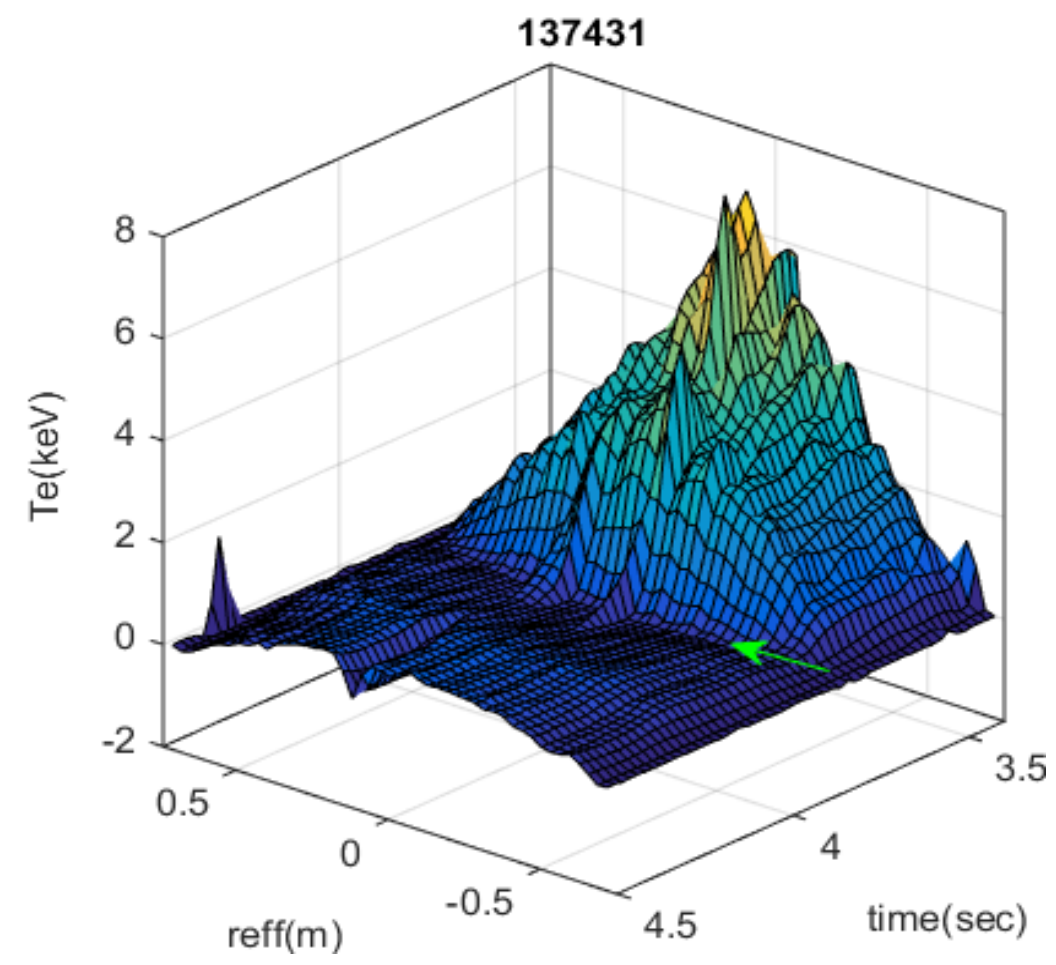

Figure 15. Surface plot of the electron temperature (z-axis) versus the minor radius (x-axis) and the time (y-axis) for \# 137431. The injection times of the pellets are indicated by an arrow.

So far, the mechanism of the rapid central density increase within $r_{\text {eff }}<0.5 \mathrm{~m}$ is not understood and could hardly be reproduced with other heating methods or pellet repetition frequencies. For more conclusions the available data basis is too sparse, nevertheless that particular example might be the key to a scenario of central particle fuelling. Certainly the positive density gradients $\left(d \mathrm{n}_{\mathrm{e}} / d \mathrm{r}>0\right.$ for $\left.\mathrm{r}_{\text {eff }}>0\right)$ in the radial range $\mathrm{r}_{\text {eff }} \approx 0.2-0.5 \mathrm{~m}$ support the central fuelling by diffusion. Nevertheless, the density increase in the plasma center occurs much faster than the density re-distribution during the plasma phase with a peaked $n_{e}$ profile.

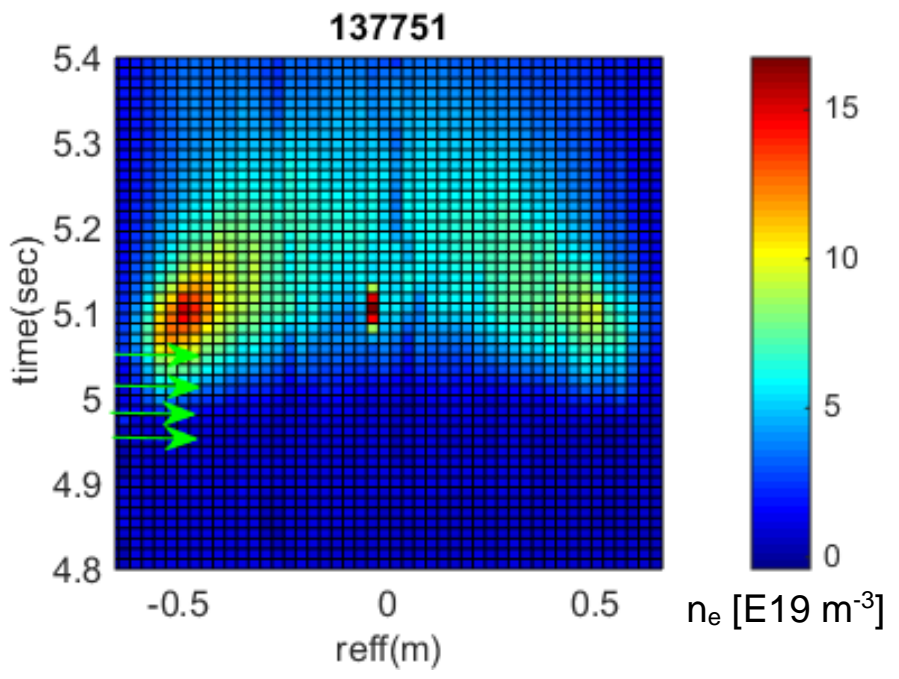


Figure 16. Contour plot of the electron density versus the minor radius (x-axis) and the time (y-axis) for \# 137751. The injection times of the pellets are indicated by arrows. The origin of the local $n_{e}$ increase in the middle of the plot is unknown, however it might real because the $T_{e}$ at the same time and location drops (see fig. 17). The side bar indicates the color code of the electron density in units of $E 19 \mathrm{~m}^{-3}$.

The third prominent example of density profile development is shown in fig. 16, again with 4 pellets and $30 \mathrm{~Hz}$ repetition frequency. The corresponding $T_{e}$ profiles are shown in fig. 17. In this case the series of pellets establishes a local density increase close to the plasma edge around $\mathrm{r}_{\mathrm{eff}} \approx 0.4-0.6 \mathrm{~m}$, forming hollow density profiles in accordance to the localized edge particle sources. Then, on a diffusive time scale, the hollow profiles relax to more peaked ones with a subsequent increase of the central density.

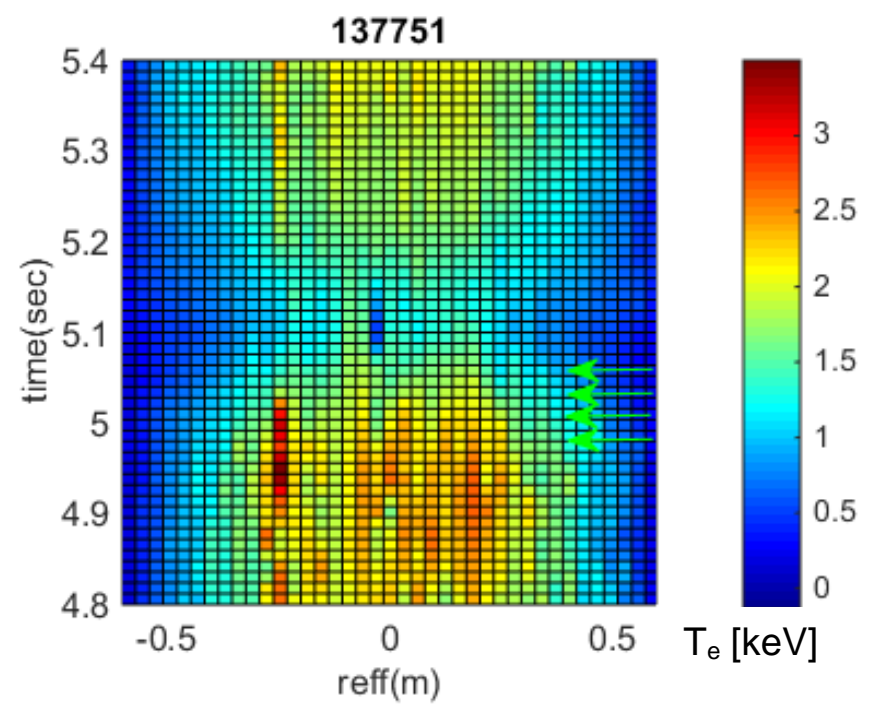

Figure 17. Contour plot of the electron temperature versus the minor radius (x-axis) and the time (y-axis) for \# 137751. The injection times of the pellets are indicated by arrows. The side bar indicates the color code of the electron temperature in units of keV.

This type of behavior is not surprising and can be understood on the basis of diffusion. It is rather typical for many of the discharges investigated and can easily be reproduced.

The most interesting case shown in fig. 14 with a fast central density increase after a hollow profile phase requires some more attention. When looking through all 100 discharges performed, the question came up which discharge conditions must be fulfilled to observe such a rapid central fuelling effect. The table I shows a list of the six discharges that showed the rapid central fuelling effect. All other 94 discharges resemble the behavior shown in the figs. 12 or 16.

\begin{tabular}{|l|l|l|l|l|l|}
\hline Shot no. & Ice isotope & Heating & Frequ. $[\mathrm{Hz}]$ & Rax [m] & $\begin{array}{l}\text { Plasma } \\
\text { isotope }\end{array}$ \\
\hline 137404 & D2 & ECRH + NBI & 90 & 3.6 & D \\
\hline
\end{tabular}




\begin{tabular}{|l|l|l|l|l|l|}
\hline 137431 & H2 & ECRH + NBI & 30 & 3.6 & D \\
\hline 137439 & H2 & ECRH + NBI & 300 & 3.6 & D \\
\hline 137499 & D2 & ECRH + NBI & 30 & 3.9 & D \\
\hline 137471 & H2 & ECRH + NBI & 90 & 3.9 & D \\
\hline 137759 & H2 & ECRH + NBI & 30 & 3.75 & D \\
\hline
\end{tabular}

Table I. List of all discharges that show a fast central fuelling effect after a hollow density profile phase. Shown are the shot numbers, the pellet ice isotope, the heating scenario, the pellet repetition frequency, the major axis position, the plasma main isotope.

These six discharges have in common that they are heated by NBI plus ECRH into a deuterium plasma, and that rather long series of 4 or 5 pellets had been performed. All other factors deviate from each other. That means that obviously neither the pellet ice isotope, nor the repetition frequency, nor the major axis position seem to affect the rapid central fuelling. This holds in principle for all other plasma parameters prior to the series of pellets. Albeit the discharges start from comparable parameters, only some of them develop that central fuelling, others do not. So far we have no explanation for that observation. Further experiments will be needed to confirm whether NBI plus ECRH heating into deuterium discharges are the prerequisites to maintain that scenario. In total 34 discharges out of the 100 were heated by NBI plus ECRH into deuterium plasmas, but the other 28 of them do not show that fast central fuelling $(<30 \mathrm{~ms})$.

\section{Discussion}

The experiments performed in LHD with different heating scenarios provide indications, that the injection of a series of pellets can be more efficient for deep particle fuelling than single pellets. Key factor seems to be the cooling of the plasma by initial pellets, thus helping later pellets in the series to penetrate deeper. Independent of the heating scenario, the scatter between the measured data points is larger than a possible systematic impact from the heating method. Future experiments with series of pellets in W7-X are presently planned to investigate in more detail the impact of the heating on the fuelling. For the experiments performed in LHD, the amount of ECRH power was only $\approx 25 \%$ compared to the NBI power. A clearer evidence is expected when purely NBI heated discharges are compared to purely ECRH heated discharges. Also purely ICRH heated plasmas might be desirable, for instance to investigate in more detail the influence of supra-thermal particles (electrons during ECRH, ions during ICRH) on the ablation process. Such experiments will also be helpful to assess the possible role of fusion products (alphas in a reactor) on the ablation and deposition. W7-X is able to provide purely ECRH heated plasmas with up to $10 \mathrm{MW}$, thus series of pellets programs have high priority for the next experimental campaign (Sept 2017 - Dec 2017) in purely ECRH heated plasmas. 
The pellets in W7-X will be smaller than those in LHD ( $2 \mathrm{~mm}$ nominal size compared to 3 $3.4 \mathrm{~mm}$ in LHD) and slower (250 m/s compared to $1000 \mathrm{~m} / \mathrm{s}$ in LHD) making the penetration more shallow in W7-X than in LHD and the plasma perturbation smaller. Therefore, it is assumed that series of pellets can be made in W7-X longer at comparable net particle fuelling rate than the series in LHD, where about 4-5 pellets seemed to be an upper limit. Pushing that limit might also help to provoke a temperature hole, if such a phenomenon will be accessible in W7-X. In addition, the very long discharges envisaged for W7-X (in the range of minutes duration) will be a good test-bed to reduce the pellet repetition frequency below the $30 \mathrm{~Hz}$ realized so far, shedding more light on the question which quantitative reduction of $T_{e}$ is required to maintain enhanced fuelling by series of pellets.

The low pellet velocity in $\mathrm{W} 7-\mathrm{X}$ is the price for the possibility to inject simultaneously from HFS and LFS, because the HFS guiding tube is strongly curved to allow for access to the inboard injection port. Besides the test of series of pellets versus single pellets, the potential of HFS launch in a large stellarator will be one of the major goals during the next experimental campaign, thus addressing some of the general issues listed in the introduction above.

Besides the reduction of $T_{e}$ after pellet injection, also the increase of plasma radiation might play a role for the question, whether a series of pellets can be prolongated without loss of plasma performance. As W7-X will be operated with a set of island divertors, the influence of the metallic wall as a source of heavy impurities might be repelled, as well as the hardly controllable impact of variable recycling fluxes as a function of the discharge history.

The issue of the discharge history affects also the possible error source of the background density transport during the series of pellets for our efficiency studies above. For that sake, only stationary background discharge conditions are chosen for the experiment (constant heating power, gas feed program, constant radiation, etc.) however the series of pellets itself will always impose a perturbation of the background discharge. The discharges chosen for our studies show a rapid decay of the density directly after the series of pellets which plays no role for us, because the corresponding density values for the calculations are taken 1-2 msec after the series of pellets. After typically $\approx 0.5-1 \mathrm{~s}$ after the last pellet of the series the density values stabilize again to constant values, indicating that the perturbation by the series of pellets is in fact only transient.

The pellet ice isotope seemed to play no role for the fuelling in a series of pellets, making hope that advantageous effects from that technique might also be applicable for the injection of deuterium/tritium pellets into fusion grade plasmas. As the next W7-X campaign will be with hydrogen as discharge working gas, and no deuterium discharges were performed before, plasmas with $\mathrm{H} /(\mathrm{H}+\mathrm{D})=1$ will be realized, making a later comparison with pure deuterium discharges more significant.

At the beginning of this paper the impact of $\mathrm{grad}-B$ induced drifts directly after the formation of the dense pellet plasmoid are briefly mentioned, because they will influence the post-pellet particle confinement and the final particle deposition in the plasma. In LHD we used always the same injection geometry from the outboard side, hence no variation of the direction of the grad- $B$ drift direction was possible.

Several types of density profile relaxation after a series of pellets could be observed, the most exciting being those of fast central particle fuelling, however without too strong central 
plasma cooling, too enhanced plasma radiation, or a plasma collapse. Emphasis will therefore be on the establishment of central particle fuelling mechanisms in W7-X even with shallow pellet penetration, and the mechanisms behind it should to be revealed with priority during every pellet program.

\section{Acknowledgments}

The authors would like to thank the LHD experiment group and the technical staff of LHD for their support of this work. This work is partly supported by the budget NIFS17ULPP004 of the National Institute for Fusion Science. This work has been carried out within the framework of the EURO-Fusion Consortium and has received funding from the Euratom research and training programme 2014-2018 under grant agreement No 633053. The views and opinions expressed herein do not necessarily reflect those of the European Commission.

\section{References}

[1] O. Motojima, H. Yamada, A. Komori, N. Ohyabu, K. Kawahata, O. Kaneko, S. Masuzaki, A. Ejri, M. Emoto, H. Funaba, et al., Physics of Plasmas, 6 (1999) 1843

[2] M. Wakatani, Stellarator and Heliotron Devices (1998), Oxford University Press, New York, Oxford

[3] C. Beidler, E. Harmeyer, F. Herrnegger, J. Kisslinger, Y. Igitkhanov, H. Wobig, Fusion Technol. 17 (1990) 148

[4] G. Grieger, W. Lotz, P. Merkel, J. Nührenberg, J. Sapper, H. Wobig, Phys. Fluids B 4 (1992) 2081

[5] H. Maassberg, C. Beidler, E. Simmet, Plasma Phys. Contr. Fusion 41 (1999) 1135

[6] P.T. Lang, C. Day, E. Fable, Y. Igitkhanov, F. Koechl, R, Mooney, B. Pegourie, B. Ploeckl, R. Wenninger, H. Zohm,, Fusion Eng. and Design 96-97 (2015) 123

[7] R. Raman, Fusion Eng. Design 83 (2008) 1368

[8] V. Mertens, M. Kaufmann, J. Neuhauser, J. Schweinzer, J. Stober, K. Buchl, O. Gruber, G. Haas, et al., Nucl. Fusion 37 (1997) 1607

[9] L.R. Baylor, S.K. Combs, C.R. Foust, T.C. Jernigan, S.J. Meitner, P.B. Parks, Nucl. Fusion 49 (2009) 085013 
[10] P.T. Lang, K. Buechl, M. Kaufmann, R.S. Lang, V.Mertens, H.W. Müller, Phys. Rev. Lett., 79 (1997) 1487

[11] R. Sakamoto et al, Rev. Sci. Instr. 84 (2013) 083504

[12] C. Suzuki, F. Koike, I. Murakami, N. Tamura, S. Sudo, H.A. Sakaue, N. Nakamura, S. Morita, M. Goto, T. Nakano et al., Physica Scripta 89 (2014) 114009

[13] R.C. Isler, Plasma Phys. Contr. Fusion 36 (1994) 171

[14] K. Narihara, I. Yamada, H. Hayashi, K. Yamauchi, Rev. Sci. Instr. 72 (2001) 1122

[15] L. Garzotti, L. Baylor, F. Koechl, B. Pégourié, M. Valovic, K.B. Axon, J. Dowling, C. Curl, et al., Nucl. Fusion 50 (2010) 105002

[16] S. Murakami, H. Yamada, A. Wakasa, M. Sasao, M. Isobe, T. Ozaki, P. Goncharov, T. Saida, et al., 19th Conf. Proc. Fusion Energy 2002, Sorrento, Italy, IAEA, (2003), EX/C5-3.

[17] A. Dinklage, R. Sakamoto, M. Yokoyama, K. Ida, J. Baldzuhn, C.D. Beidler, S. Cats, K.J. McCarthy et al., Nucl. Fusion 57 (2017) 066016

[18] R. Sakamoto, J. Miyazawa, H. Yamada, S. Masuzaki, A. Sagara, FFHR Design group, Nucl. Fusion 52 (2012) 083006 